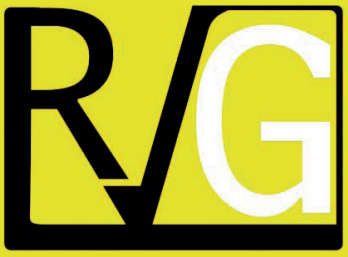

Julio - Septiembre, 2021

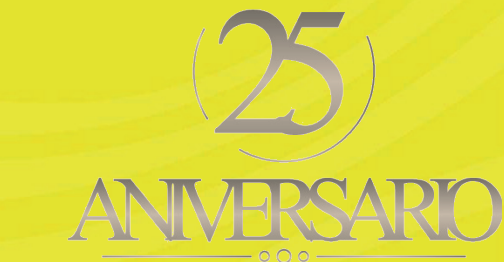

ANMERSARO
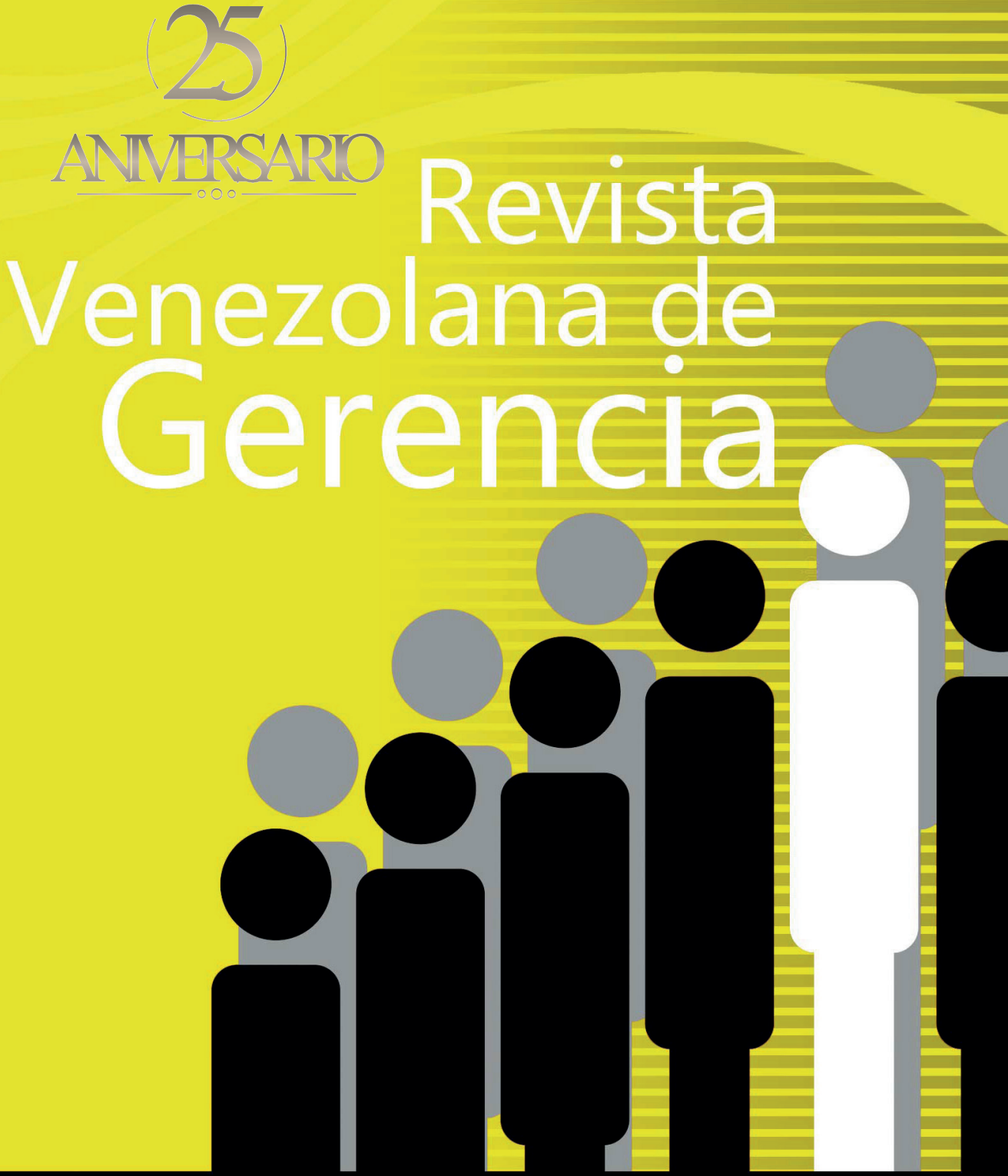

UNIVERSIDAD DEL ZULIA (LUZ)

Facultad de Ciencias Económicas y Sociales Centro de Estudios de la Empresa

ISSN 1315-99

Esta obra está bajo una licencia de Creative Comm Reconocimiento-NoComercial-Compartirlgual 3.0 Unpo http://creativecommons.org/licenses/by-nc-sa/3.0/deed.es 
COMO CITAR: Araujo-Robles, E. D., Díaz Espinoza, M., y Díaz Mujica, J. Y. (2021). Dinámica familiar en tiempos de pandemia (COVID -19): comunicación entre padres e hijos adolescentes universitarios. Revista Venezolana de Gerencia (RVG), 26(95), 610-628. https://doi. org/10.52080/rvgluz.27.95.11
Universidad del Zulia (LUZ)

Revista Venezolana de Gerencia (RVG)

Año 26 No. 95 Julio-Septiembre 2021, 610-628

ISSN 1315-9984 / e-ISSN 2477-9423

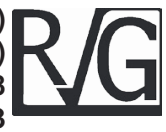

\title{
Dinámica familiar en tiempos de pandemia (COVID -19): comunicación entre padres e hijos adolescentes universitarios
}

\author{
Araujo-Robles, Elizabeth Dany* \\ Díaz Espinoza, Maribel** \\ Díaz Mujica, Juana Yris ${ }^{\star \star *}$
}

\section{Resumen}

Los cambios ocurridos, producto de la nueva situación mundial, han provocado diversas transformaciones en los estilos de vida, entre los que se destaca la convivencia en los hogares a partir del confinamiento obligatorio. El objetivo del trabajo fue analizar la dinámica familiar en tiempos de pandemia (COVID -19), comparando los procesos comunicacionales entre padres e hijos adolescentes universitarios de Piura y Lima. El estudio fue descriptivo comparativo en una muestra no probabilística por conveniencia de estudiantes provenientes de una universidad de Lima $(n=109)$ y otra de Piura $(n=107)$. Se aplicó la Escala de Comunicación Padres-Adolescente versión universitaria, adaptada en Lima (Araujo, et al. 2018). Los resultados indican que tanto en el grupo de Piura como en el de Lima, el nivel de comunicación en general con ambos padres es similar, pero dichos niveles son más elevados en el grupo de Piura. Como conclusión,

* Doctora en Psicología por la UNFV, Investigadora Renacyt P0059497, Coordinadora de la Unidad de Investigación Ciencia y Tecnología de la Facultad de Psicología de la Universidad Peruana Cayetano Heredia, Editora de la Revista Psicológica Herediana. Coordinadora General de Tutoría Psicológica de la Universidad Nacional de Ingeniería Lima_Perú. ORCID: https://orcid.org/0000-0002-9875-6097, correo: elizabeth.araujo.r@upch.pe

** Doctora en Administración de la Educación por la Universidad César Vallejo, Magíster en Docencia Universitaria y Licenciada en Obstetricia, por Univ. Cesar Vallejo. Docente de pregrado de la Universidad Tecnológica del Perú. Docente de la Escuela de Posgrado de la Universidad Cesar Vallejo Piura - Perú de la Maestría en Gestión de los Servicios de la Salud. ORCID: https://orcid.org/0000-0001-5208-8380, correo: c20937@utp. edu.pe

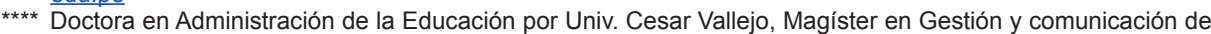
Entidades sociales y solidarias por Univ. Abad Oliba de Barcelona, Magister en Gestión y Docencia educativa por Univ. Cesar Vallejo, Licenciada en Obstetricia por Univ. San Martín de Porres. Docente de la escuela de Posgrado de la Universidad Cesar Vallejo-Perú. ORCID: https://orcid.org/0000-0001-8268-4626. jdiazmu@ ucvirtual.edu.pe 
se obtuvo que en ambos grupos se aprecia un mayor nivel de apertura frente a la madre y mayor nivel de problemas de comunicación con el padre.

Palabras clave: adolescentes; comunicación familiar; Covid-19; padres; universitarios.

\title{
Family dynamics in times of pandemic (COVID -19): communication between parents and university adolescents
}

\begin{abstract}
The changes that have occurred, as a result of the new world situation, have caused various transformations in lifestyles, among which the coexistence in homes from compulsory confinement stands out. The purpose was to compare levels of communication between parents and their sons and daughters, who are university students in Lima and Piura (Perú) in times of Covid-19 pandemic. This study was descriptive and comparative in a not probabilistic convenience sample of students from a university from Lima $(n=109)$ and other from Piura $(n=107)$. They were administered the Barnes and Olson's Scale of Parent-Adolescent Communication, in an adapted version for university students by Araujo et al. (2018). The results indicate that levels of communication were higher in Piura and the openness in communication with mother was higher than with father, and levels of communication problems were higher with fathers than with mothers. Conclusión; In both groups, there is also a greater level of openness towards the mother and a greater level of communication problems with the father.
\end{abstract}

Key words: adolescents; communication; Covid-19; parents; university students.

\section{Introducción}

Iniciando el 2020 comenzó la pandemia por covid-19, la enfermedad que es trasmitida por el coronavirus conocido como SARS-Cov-2. Pocas veces la humanidad había enfrentado una emergencia sanitaria de gran impacto, la última referencia de una situación similar sucedió hace 100 años atrás con la llamada gripe española. El contagio se extendió a nivel global y ha forzado a los gobiernos a adoptar medidas drásticas de contención, incluyendo cuarentenas masivas, obligando así a millones de personas a permanecer en sus casas y al cierre o paralización de una gran cantidad de actividades y de empresas (Lippi et al. 2020). Esas medidas se han prolongado por varios meses e incluso, en algunas partes, después de que fueron revocadas, se han tenido que restablecer debido al rebrote de la enfermedad. 
En un contexto actual de pandemia, en el que se incrementan las reacciones de estrés y ansiedad en general (e. g., Apaza et al. 2020; Balluerka et al. 2020; Wang et al. 2020), el soporte brindado por una buena comunicación con sus padres puede ser de gran importancia para que los adolescentes se adapten de manera sana a las circunstancias, ellos generan escenarios que le permita a sus hijos satisfacer sus necesidades y lograr un crecimiento adecuado (Velásquez y Salom, 2008).

La búsqueda de autonomía por parte de los adolescentes y la necesidad de los padres por mantener su autoridad (Guzmán \& Pacheco, 2014), se traduce por ambas partes en las diversas estrategias de comunicación que existen en el interior de la familia, en momentos como los actuales en que se incrementa la probabilidad de desajustes emocionales en las personas.

Una serie de estudios han enfocado la calidad de la comunicación del adolescente con cada una de las figuras parentales durante el período de pre-pandemia. Por ejemplo, en universitarios colombianos se encontró un nivel promedio de diálogo con la madre y con el padre, lo que indica que pueden existir dificultades en la comunicación, siendo ésta poco eficaz, excesivamente crítica o negativa. En cuanto al nivel de dificultades con los progenitores, se evidencia que es mayor con el padre, centrando la comunicación en aspectos como la resistencia a compartir información y afecto o estilos negativos de interacción (Crespo, 2011).

Las familias que muestran una mayor adaptabilidad en su funcionamiento familiar tienen a su vez mayor vinculación emocional entre sus miembros y capacidad para entenderse en función de las demandas.
En un estudio se encontró que los adolescentes tenían una comunicación más abierta con la madre, con la cual sentían que podían comunicarse sin incomodidad y libremente, percibiendo que recibían atención y que había confianza, empatía, facilidad y libertad para expresar emociones, pensamientos y problemas, lo que facilitaba la cohesión familiar (Garrido et al. 2019).

Por su parte Garcés et al. (2020), indican que la calidad de la comunicación entre padres y adolescentes influye en la conducta de éstos; específicamente señalan que cuando la comunicación familiar es abierta disminuye la victimización y la probabilidad de que los adolescentes adopten comportamientos agresivos; en cambio, cuando es ofensiva la probabilidad de victimización aumenta. Otros estudios indican que hay un nexo entre la comunicación con los padres, la actitud positiva hacia la autoridad institucional, el malestar psicológico y la victimización en el adolescente (Castañeda et al. 2019). También se encontraron relaciones positivas y significativas directas entre las emociones positivas, las relaciones percibidas en el entorno escolar, las conductas prosociales y la comunicación basada en el afecto de ambos padres (Ruvalcaba et al. 2018).

La comunicación en general, como objeto de estudio, desde diferentes abordajes es un factor de gran importancia en el desarrollo de la sociedad y del individuo, de allí la importancia de investigar cómo se desarrolla especialmente entre los padres y sus hijos adolescentes (Santos, 2019). Sin embargo, la revisión arriba presentada evidencia la falta de estudios respecto a la comunicación intrafamiliar en tiempos de inmovilidad obligatoria. Resulta importante conocer 
los patrones de comunicación entre padres y sus hijos adolescentes en un tiempo de crisis como el actual. De acuerdo con ello, el objetivo general del presente estudio es analizar la dinámica familiar en tiempos de pandemia (COVID -19), comparando los procesos comunicacionales entre padres e hijos adolescentes universitarios de Lima y Piura, dos ciudades importantes de la costa del Perú.

\section{Dinámica familiar en tiempos de pandemia}

La pandemia en sí y las medidas
adoptadas para su contención
provocan consecuencias de diversa
gravedad en el estado emocional de
las personas. Estas reacciones no sólo aparecen, comprensiblemente, entre los profesionales sanitarios que deben atender pacientes afectados por covid-19 (Kang et al. 2020), sino también entre el público en general (Huarcaya, 2020; Zhou, et al. 2020a). No sólo la presencia en sí de la pandemia y de la amenaza del contagio provocan estrés, ansiedad y otros síntomas, sino también el confinamiento obligatorio mismo y las circunstancias asociadas a él como la pérdida financiera, limitaciones de aprovisionamiento y de atención médica, etc. (Brooks et al. 2020).

La alteración de la rutina puede llegar a provocar problemas de salud mental como frustración por la sensación de dejar de hacer algo, ansiedad y temores (Hsieh et al. 2020; Koushik, 2020; Pastor, 2020; Serafini et al. 2020), así como otros factores psicosociales relacionados con el estrés y depresión (Apaza et al. 2020) además, la pérdida del contacto diario con los colegas y amigos causa frustración y apatía (Brooks et al. 2020). El confinamiento genera la aparición de diversas manifestaciones (Dorrance et al. 2020), como la frustración por actividades negadas, una sensación de falta de libertad, la ausencia física de los amigos, entre otros; además de otros efectos sociales y económicos, como pueden ser la pérdida de trabajos, de emprendimientos, etc. También se ha demostrado que entre los efectos psicológicos asociados a la pandemia se hallan los trastornos psicóticos que pueden llevar a los suicidios (Daniels \& Rittenour, 2020). De esta manera, las familias enfrentan un mayor riesgo para su bienestar, como consecuencia del confinamiento y en general, las diversas incertidumbres que se derivan de la actual situación sanitaria (Prime et al. 2020).

Los efectos de la pandemia y del confinamiento influyen en la dinámica de la familia, pero esa dinámica, a su vez también influye en la adaptabilidad de los miembros familiares frente a esta crisis. La dinámica que se vive al interior de las familias está relacionada a los roles que cada integrante desempeña, así como con el ambiente que en ella se presente, de modo que los roles familiares funcionales adecuados inciden de manera positiva en la vida de las familias (Delfín et al. 2020).

En el contexto de la actual pandemia surgen cuestiones de interés respecto al desarrollo de la dinámica familiar. El confinamiento social como medida de protección frente al contagio ha ocasionado que las familias convivan las 24 horas del día durante varios meses (Brooks et al. 2020), hecho que no ha sido común en los últimos tiempos, los cuales se habían caracterizado más bien por lo agitado de las jornadas familiares.

Las consecuencias del confinamiento sobre la dinámica familiar están ampliamente determinadas por la 
calidad de las relaciones familiares que existían previamente, como también por la reacción de cada integrante de la familia a la crisis sanitaria y al confinamiento. Por ejemplo, el confinamiento no necesariamente implica una mejora en la calidad de las relaciones entre los miembros del grupo familiar. Por el contrario, es posible que, en algunos casos, conflictos y desavenencias previamente existentes, incluso la violencia, se incrementen (Bradbury-Jones \& Isham, 2020; Campbell, 2020; Usher et al. 2020).

Las consecuencias emocionales del confinamiento y de la pandemia en sí, se evidencian en las familias con molestias, ira, frustración y preocupaciones diversas, como, por ejemplo, por el modo de subsistencia y por la variación de lo presencial al teletrabajo (Broche-Pérez, FernándezCastillo y Reyes, 2020) aunque no todos pueden acceder a este cambio. En el Perú, sólo el $16 \%$ de las personas en edad de trabajar tiene un trabajo estable o están en planilla y la mayoría de las familias se sostienen económicamente de manera independiente. Ello ya constituía una situación difícil en tiempos previos a la pandemia, agravándose más aún durante la cuarentena, durante la cual muchas actividades económicas se paralizan (CEPAL, 2020).

En circunstancias normales, la calidad de la comunicación familiar tiene implicaciones importantes para el desarrollo o no de determinados problemas conductuales en los adolescentes (Kapetanovic et al. 2020; Pinquart, 2017). Una buena comunicación con sus padres puede contribuir al desarrollo de algunas estrategias de afrontamiento al estrés en los adolescentes (Simpson et al. 2020). Si todo ello es cierto en circunstancias normales, lo es más aún en el contexto sanitario actual, en la medida que una efectiva comunicación acerca de los riesgos ocasionados por la presencia de la pandemia puede contribuir a un mejor bienestar para la familia y los niños y adolescentes que forman parte de ella (Dalton et al. 2020). Si bien es cierto que aun la madre es la figura más presente en los hogares, también se observa que últimamente la comunicación entre papá e hijos resulta ser muchas veces influyente para los jóvenes (Raimundi et al. 2017).

En el presente estudio se adoptó el modelo que fue propuesto por Olson et al. (1979), para describir las dimensiones básicas del funcionamiento de la pareja y de la familia: la cohesión y la flexibilidad. Posteriormente, se adicionó la dimensión de comunicación. La cohesión se define como el "vínculo emocional que existe entre los miembros de la pareja o la familia" (Olson et al. 2019:201-202), mientras que la flexibilidad consiste en "la cantidad de cambio en el liderazgo, los roles y las reglas". Los mismos autores mencionan que la cohesión y la flexibilidad son curvilíneos (los niveles más bajos y más altos son considerados problemáticos), en tanto que la comunicación es lineal: a un nivel más elevado, es mejor el funcionamiento.

La combinación de los diferentes niveles de cohesión y flexibilidad da lugar a varios tipos de familia o pareja, de los cuales algunos son considerados balanceados (cuando se combinan niveles intermedios de cohesión y de flexibilidad) y otros más bien son etiquetados como no balanceados (cuando se combinan niveles bajos o altos de una dimensión con niveles altos o bajos de la otra). Una familia o pareja caótica-disgregada es la que 
resulta de la combinación de niveles altos de flexibilidad y niveles bajos de cohesión. Por último, la comunicación es considerada como la dimensión que permite el cambio de la familia o pareja a través de las dos primeras dimensiones con el fin de afrontar mejor las circunstancias situacionales o del propio proceso evolutivo de la pareja o la familia (Olson \& Gorall, 2003).

De acuerdo con lo planteado por diversos investigadores (e. g., Gomes \& Gouveia-Pereira, 2019; Turkdogan et al. 2019), el modelo plantea tres hipótesis fundamentales: (a) las familias y las parejas balanceadas son más "funcionales" (i. e. felices o exitosas) que las no balanceadas; (b) las familias y las parejas más balanceadas tienen mejor comunicación que las no balanceadas y (c) las familias o parejas balanceadas tienen una mejor capacidad para modificar sus niveles de cohesión y de flexibilidad a fin de adaptarse a situaciones de estrés o a los cambios. La segunda de estas hipótesis, que es directamente relevante al tema de la comunicación, es apoyada por la evidencia cuando se trata de la calidad de la comunicación padres-adolescentes, tal como es percibida por los padres; sin embargo, no cuando dicha variable es evaluada desde la perspectiva de los adolescentes. Además, los padres y sus hijos adolescentes perciben de diferente manera la calidad de la comunicación entre ellos, y esas diferencias pueden estar asociadas a determinados problemas psicológicos en los adolescentes (Kapetanovic \& Boson, 2020).

La dinámica de la comunicación familiar presenta diversos matices tal como lo exponen algunos estudios que indicaron que la cohesión y adaptabilidad son factores preponderantes en la funcionalidad familiar (Rivera \& Cahuana, 2016); por otro lado, la falta de cohesión y una rigidez en la adaptabilidad, además de insuficiente diálogo efectivo, por el poco tiempo compartido, provocan que cada miembro de la familia reaccione de modo singular frente a las crisis (Villavicencio \& Villarroel, 2017).

Lacohesión, definidacomolos lazos de unión emocional que se construyen en la familia, así, el afecto, el grado de aproximación entre los miembros, hacen que las familias convergen en unidad (Moretti et al. 2020). Las relaciones intrafamiliares se definen por la cohesión que muestran; existen desde las desligadas, hasta las enmarañadas, lo cual, no resultan adecuados para un buen funcionamiento, por ser los extremos. Por otro lado, Gouveia et al. (2020) indico que la cohesión mapea el equilibrio de la conexión emocional en el sistema familiar y la separación entre el individuo y la familia. En el seno de las familias se producen genuinas relaciones Interpersonales (Delfín, et al. 2020) estableciéndose como dinámica familiar, el equilibrio de las mismas que hacen que en los momentos críticos se mantenga la unidad y el deseo de salir adelante.

La emergencia sanitaria permite que la adaptabilidad que es la capacidad que tiene el grupo familiar para reorganizarse de cara a la nueva situación aflore (Gouveia, et al. 2020). Es necesaria una respuesta al estrés del momento, en la cual surgen diversos temas, por un lado, el confinamiento, el temor al contagio, la situación económica, y otras, que podrían producir ansiedad y pánico en algunos casos. Sin embargo, está fuera de toda duda que la comunicación entre los padres y sus hijos adolescentes está muy relacionada con diversos indicadores de salud mental en 
estos últimos, como, por ejemplo, el uso problemático de internet (Liu et al. 2019), el autoconcepto (Van Dijk et al. 2014) y los indicadores de estrés postraumático después de una catástrofe natural (Zhou, et al. 2020b).

\section{Situación de los jóvenes en tiempo de pandemia}

Respecto a los adolescentes y jóvenes la situación actual de pandemia y confinamiento se convierte en una preocupación de los padres o tutores, ya que los jóvenes no se encuentran ajenos a la problemática relacionada con la pandemia. En realidad, experiencias anteriores muestran que los niños y adolescentes se ven significativamente afectados en su salud mental a causa del confinamiento (Espada et al. 2020). Lozano et al. (2020) verificaron que el confinamiento generó gran impacto psicológico y académico en los universitarios españoles, lo cual condujo a los autores a proponer una mejora en la resiliencia desde las aulas universitarias. Evidencias recientes en el contexto de la actual pandemia, también revelan efectos emocionales negativos en niños y adolescentes (Duan et al. 2020).

Normalmente, los jóvenes pasan gran parte de su tiempo entre los estudios y los amigos; además, muchos de ellos también trabajan. Es frecuente que los jóvenes encuentren soporte en sus pares en sus momentos de estrés por lo que es comprensible que el cierre de los centros educativos tenga también un efecto negativo en niños y adolescentes (Lee, 2020; Kilincel et al. 2020, Zhou, Zhang et al. 2020a). Por ejemplo, los adolescentes muestran también preocupación respecto del futuro de su vida escolar y social (Kilincel et al. 2020). Los adolescentes sienten una gran necesidad de establecer relaciones interpersonales más allá del medio familiar inmediato y, como observan Imran et al. (2020), el confinamiento puede, en el mejor de los casos, hacerlos sentir aburridos, nostálgicos y frustrados, y en el peor, desencadenar manifestaciones más severas de desajuste emocional.

Algunos estudios comparativos son reveladores en cuanto al impacto de las medidas de confinamiento sobre los adolescentes. Por ejemplo, Saurabh y Ranjan (2020) comprobaron en India que los adolescentes en cuarentena experimentaronmayoresnivelesdeestrés que los no confinados. Las principales reacciones emocionales observadas fueron miedo, preocupaciones y desesperanza. Nuevamente, estos autores enfatizaron la importancia de la compañía de los pares para el bienestar de los adolescentes, compañía que se ve limitada durante el confinamiento.

No solo la pandemia misma o el confinamiento tienen efectos adversos sobre los adolescentes, sino también otros factores asociados a la actual situación sanitaria. Por ejemplo, la excesiva exposición a información, frecuentemente alarmista y poco fundamentada, es un factor de estrés para niños y adolescentes (Centre for Disease Control, 2020; Dalton et al. 2020). El aislamiento social obligatorio conlleva un incremento el uso de las redes sociales como medio de contacto, lo cual, a su vez, puede implicar un aumento en el riesgo de ser víctimas de ciberbullying o de exposición a contenidos inapropiados, como indican Imram et al. (2020), quienes también alertan acerca de la probabilidad incrementada de que niños y adolescentes sufran abuso, descuido o explotación al encontrarse en confinamiento obligatorio. 
Sin embargo, siendo la familia la entidad encargada de mantener la seguridad y protección de los hijos (Torales et al. 2020), es necesario que los integrantes del grupo familiar se comuniquen mejor $y$ de ese modo, busquen soluciones ante las circunstancias actuales. Todo ello involucra, por supuesto, pautas saludables de conducta individual y de interacción entre los miembros familiares, algo que, en algunos casos, es difícil de mantener antes las circunstancias de incertidumbre y tensión provocadas por la crisis sanitaria

\section{Consideraciones metodológicas}

En cuanto a la metodología, el estudio fue de diseño descriptivo comparativo, no experimental de campo transaccional, con una muestra de 216 adolescentes universitarios, de ambos sexos, que viven con sus padres y cursan los dos primeros años de estudios. Dichos estudiantes tenían edades entre 18 y 21 años ( $M=19.3$; $D E=2.1)$ y procedían de dos universidades, una ubicada en la ciudad de Lima (capital del país) y la otra en la ciudad de Piura, el criterio de inclusión fue la condición de vivir con ambos padres y la participación fue voluntaria. Para el cálculo del tamaño de muestra se tuvo en cuenta criterios de tamaño de efecto y de potencia de la prueba (Polit \& Beck, 2012). Se asumió un tamaño de efecto mediano (alrededor de $r=.35$ ) para estudios de diferencias de medias. Considerando un nivel de significación de .05 y una potencia de prueba de .99, se estimó el tamaño de muestra en mínimo 160 , teniendo como criterios de inclusión la condición de vivir con ambos padres y la participación voluntaria.
Se aplicó la Escala de Comunicación Padres-Adolescente versión universitaria, ECPA-U (Araujo et al. 2018), mide la calidad de la comunicación padres-adolescente por medio de las escalas de apertura de la comunicación y problemas de comunicación. Los ítems se responden mediante una escala tipo Likert con cinco alternativas de respuesta, las cuales permiten expresar el grado de acuerdo del participante con lo expuesto en el ítem. Cada uno de estos ítems describe alguna conducta o situación relevante a la calidad de la comunicación. Las puntuaciones de los ítems de apertura son directas, en tanto que las de problemas deben invertirse. Ambas puntuaciones pueden sumarse, dando como resultado una puntuación global en calidad de la comunicación.

Se analizaron los datos usando las medidas de tendencia central y se analizaron las diferencias según lugar de procedencia, empleando la $U$ de MannWhitney, después de la verificación de los resultados de la prueba de ajuste a la normalidad, que se efectuó para la muestra total y los subgrupos según la procedencia.

\section{Comunicación entre padres e hijos adolescentes universitarios en las ciudades peruanas de Lima y Piura}

Para el cálculo del tamaño de muestra se tuvo en cuenta criterios de tamaño de efecto y de potencia de la prueba (Polit \& Beck, 2012). Se asumió un tamaño de efecto mediano (alrededor de $r=.35$ ) para estudios de diferencias de medias. Considerando un nivel de significación de .05 y una potencia de prueba de .99 , se estimó el tamaño 
de muestra mínimo de 160. Se aplicó la Escala de Comunicación PadresAdolescente versión universitaria, ECPA-U (Araujo et al. 2018). La convocatoria fue abierta mediante mensajes a los correos electrónicos de los estudiantes y la participación fue voluntaria. Para el análisis de datos, se calcularon las medidas de tendencia central para la muestra total y para cada subgrupo (Lima y Piura), también se realizaron las pruebas de bondad de ajuste para evaluar si los datos presentan o no distribución normal y finalmente se empleó la prueba $U$ de Mann-Whitney para realizar las comparaciones según lugar de procedencia.

En la Tabla 1 se aprecian los niveles de comunicación con ambos padres en la muestra total. En términos globales, el nivel de comunicación es casi el mismo con ambas figuras paternas, sin embargo, se aprecian diferencias notables en la comunicación frente a una u otra de dichas figuras, cuando se analizan las subescalas por separado. El nivel de apertura con la madre es más alto que con el padre, mientras que el nivel de problemas de comunicación con la madre es más bajo que con el padre. Estos primeros resultados indican que la puntuación total no es siempre un indicador confiable de las posibles diferencias en la calidad de la comunicación en relación con cada figura parental. Los patrones de comunicación con el padre y con la madre son inversos entre sí, pero dichos patrones pueden quedar ocultos en las puntuaciones totales.

\section{Tabla 1}

\section{Niveles de comunicación padres-adolescentes en la muestra total}

\begin{tabular}{lccccl}
\hline & $\mathrm{N}$ & $\mathrm{M}$ & $\mathrm{DE}$ & $\mathrm{Pc}$ & \multicolumn{1}{c}{ Nivel } \\
\hline Problema padre & 216 & 25,81 & 7,629 & 55 & Promedio \\
Apertura padre & 216 & 33,07 & 8,190 & 75 & alto \\
Apertura madre & 216 & 36,88 & 7,279 & 65 & tendencia alta \\
Problema madre & 216 & 21,84 & 8,014 & 25 & Bajo \\
Comunicación padre & 216 & 58,88 & 8,686 & 80 & Alto \\
Comunicación madre & 216 & 58,72 & 9,217 & 55 & Promedio \\
\hline
\end{tabular}

Nota: $\mathrm{n}=$ tamaño de muestra; $\mathrm{M}=$ media; $\mathrm{DE}=$ desviación estándar; $\mathrm{pc}=$ rango percentil

Fuente: elaboración propia

Estos resultados son positivos ya que hasta cierto punto indicarían que la calidad de la comunicación hacia ambas figuras paternas parece ser similar, incluyendo el hecho de que con ambos el nivel de apertura es mayor que el nivel los problemas de comunicación. Todo ello implica diversas consecuencias positivas; por ejemplo, como señalan Raimundi et al. (2017), una comunicación positiva (más abierta y con menos problemas) con ambos padres predice el disfrute y el tiempo dedicado a actividades estructuradas, como el 
deporte o las actividades artísticas. Sin embargo, es necesario también tener en cuenta que, en algunos casos, el nivel de comunicación con cada figura paterna puede tener efectos diferenciados según se trate del padre o de la madre. Según Simpson et al. (2020) el desarrollo de estrategias de afrontamiento centradas en la emoción en adolescentes varones, estuvo correlacionado positivamente con el nivel de comunicación con sus madres, pero negativamente con la comunicación con los padres.

Al respecto, Hernández et al. (2017) mostraron que los adolescentes de edad media poseen una comunicación positiva con ambos progenitores, alto nivel de cohesión y se sienten satisfechos con sus familias mientras que los adolescentes tardíos percibieron límites en la comunicación con ambos padres, y se sienten poco satisfechos con sus familias. Sin embargo, los adolescentes de edad tardía que participaron en el presente estudio parecen mostrar en general un buen nivel de comunicación con sus padres, especialmente con la madre. Las diferencias observadas en el nivel de comunicación con cada figura paterna parecen reflejar un patrón frecuente en muchos otros contextos culturales, donde la comunicación abierta con la madre se ubicó principalmente en el nivel alto, en tanto que con el padre no había la misma confianza y libertad para expresarse y hablar (Garrido \& Reyes, 2019).

En particular la comunicación con la madre es de gran importancia para el adolescente, para Castañeda et al. (2019) la comunicación cuando es ofensiva con la madre es más determinante en la percepción de los adolescentes en la calidad de la comunicación con sus padres. Una mejor calidad de la comunicación en particular con la madre participa como un factor predictor en la capacidad del adolescente de gestionar emociones positivas (Ruvalcaba et al. 2018). En tanto que, un mayor nivel de dificultades de comunicación con el padre, genera resistencia a compartir información y por lo tanto un estilo negativo de interacción (Cudris et al. 2020), tabla 2.

Tabla 2

Niveles de comunicación padres-adolescente en los grupos de Lima y de Piura

\begin{tabular}{lllllll}
\multicolumn{1}{c}{ Adolescentes de Lima } & N & M & DE & Pc & Nivel \\
\hline Problemas padres & 109 & 23,98 & 6,763 & 40 & Promedio \\
\hline Apertura padre & 109 & 32,66 & 8,329 & 80 & Alto \\
\hline Apertura madre & 109 & 36,76 & 6,291 & 65 & Tendencia alta \\
\hline Problemas madres & 109 & 19,65 & 6,706 & 15 & Bajo \\
\hline Comunicación padre & 109 & 56,64 & 6,918 & 60 & Promedio \\
\hline Comunicación madre & 109 & 56,41 & 5,445 & 45 & Promedio \\
\hline
\end{tabular}


Araujo-Robles, Elizabeth Dany; Díaz Espinoza, Maribel; Díaz Mujica, Juana Yris

Dinámica familiar en tiempos de pandemia (COVID -19): comunicación entre padres e hijos adolescentes universitarios

\section{Cont... Tabla 2}

\begin{tabular}{llllll}
\hline \multicolumn{1}{c}{ Adolescentes de Piura. } & N & M & DE & Pc & Nivel \\
\hline Problemas padres & 107 & 27,68 & 8,029 & 65 & Tendencia alta \\
\hline Apertura padre & 107 & 33,49 & 8,063 & 75 & Alta \\
\hline Apertura madre & 107 & 36,99 & 8,193 & 65 & Tendencia alta \\
\hline Problemas madres & 107 & 24,07 & 8,629 & 50 & Promedio \\
\hline Comunicación padre & 107 & 61,17 & 9,687 & 85 & Alta \\
\hline Comunicación madre & 107 & 61,07 & 11,448 & 75 & Alta \\
\hline
\end{tabular}

Fuente: elaboración propia

La Tabla 2 muestra un patrón de resultados similar en los grupos de Lima y Piura, lo que se refleja en el patrón observado en la muestra total. Se aprecia que tanto en el grupo de Piura como en el de Lima, el nivel de comunicación en general con ambos padres es similar, pero dichos niveles son más elevados en el grupo de Piura. Por otro lado, en ambos grupos también se aprecia un mayor nivel de apertura frente a la madre y mayor nivel de problemas de comunicación con el padre. Respecto de los universitarios de Lima, la puntuación global es casi la misma para madre y padre, sin embargo, cuando se analizan las sub-escalas, se aprecia claramente un mejor nivel de comunicación con la madre. La puntuación en problemas de comunicación es más baja y la de apertura más alta respecto de la madre que del padre.

En el caso de los estudiantes de Lima, las diferencias frente a cada figura parental se aprecian cuando se analizan los resultados a nivel de subescalas. Puede verse que la puntuación en problemas de comunicación con la madre es más baja que la del padre, pero la puntuación en apertura es más alta en la madre, cabe indicar, además, que estas puntuaciones globales se debe a una mayor puntuación en la escala de problemas de comunicación, la cual es más elevada para ambas figuras parentales en el grupo de Piura, en tanto que la puntuación en apertura es casi la misma que la del grupo de Lima. Un mejor nivel de comunicación en general con la madre puede tener también un impacto positivo incluso en aquellos episodios en los que parece haber desacuerdos y conflicto con la figura materna. Caughlin y Ramey (2005) observaron que las madres son más exigentes al momento de la negociación que los padres, lo cual puede repercutir en la respuesta conductual del hijo (Pérez \& Alvarado, 2015). La mayor conflictividad con la madre puede radicar en que chicos y chicas pasan más tiempo con ella y a que la mayor parte de los temas de conflicto tienen que ver con la vida cotidiana en la que, la madre tiene un protagonismo muy especial (Rodrigo et al. 2005).

Las madres pueden suponer que siempre están atentas a las necesidades y las demandas de los hijos, pero éstos pueden tener una postura más crítica al respecto, sobre todo cuando se encuentran en la etapa de la adolescencia y están formando 
criterios y juicios propios acerca del funcionamiento familiar (Barneveld, 2012). Por lo tanto, los adolescentes discuten más con sus madres que con sus padres (Berbasé et al. 2001), pero esto se debe, justamente, a que culturalmente, las madres muestran más cercanía física y emocional con sus hijos (Oliva et al. 2002), por lo tanto, los chicos pueden intentar convencerlas de lo beneficioso de sus ideas, así como de sus necesidades. Es decir, en situaciones de posible conflicto con la autoridad parental, los adolescentes sienten más confianza de poder cuestionarla expresándose frente a la figura materna.

Los resultados de la Tabla 3 indican que las variables de comunicación padres-adolescentes se distribuyen de manera no normal en ambos grupos (excepto en el caso de problemas de comunicación en el grupo de Piura), por lo que la comparación de las puntuaciones entre ambos grupos se realizó mediante la prueba $U$ de Mann-Whitney.

\section{Tabla 3}

\section{Prueba de Kolmogorov-Smirnov de normalidad de la distribución de las puntuaciones de las variables de comunicación padres- adolescentes}

\begin{tabular}{lllllll}
\hline & PCP & AP & PCM & AM & CP & CM \\
\hline Grupo: total & & & & & & \\
\hline Estadístico & .052 & .147 & .062 & .121 & .115 & .134 \\
\hline Sig. & .200 & $<.001$ & .045 & $<.001$ & $<.001$ & $<.001$ \\
\hline Grupo: Piura & & & & & & \\
\hline Estadístico & .083 & .164 & .121 & .118 & .151 & .141 \\
\hline Sig. & .064 & $<.001$ & .001 & .001 & $<.001$ & $<.001$ \\
\hline Grupo: Lima & & & & & & \\
\hline Estadístico & .107 & .147 & .074 & .124 & .125 & .117 \\
\hline Sig. & .004 & $<.001$ & .176 & $<.001$ & $<.001$ & .001 \\
\hline
\end{tabular}

Nota: $\mathrm{PCP}=$ problemas de comunicación padre; $\mathrm{AP}=$ apertura de comunicación padre; $\mathrm{PCM}=$ problemas de comunicación madre; $\mathrm{AM}=$ apertura de comunicación madre; $\mathrm{CP}$ = comunicación total padre; $\mathrm{CM}=$ comunicación total madre.

Fuente: elaboración propia

En la Tabla 4 se presenta los resultados de las comparaciones de las puntuaciones en la escala general y en ambas subescalas frente a cada figura paterna en los grupos de Piura y Lima. Se observa que la puntuación general de comunicación en los estudiantes de
Piura es significativamente más alta que la correspondiente a los de Lima. Por otro lado, en cambio, se puede apreciar que no hay diferencia significativa entre los grupos de Piura y de Lima en relación con la apertura, ni en el caso de la madre ni del padre. De ello se puede inferir que 
en ambos grupos se manifiesta con igual intensidad la facilidad percibida para expresar sus intereses, sentimientos y metas.

Finalmente, se observa también que las puntuaciones en problemas de comunicación, tanto con el padre como con la madre, son significativamente más altas en el grupo de Piura. Estos resultados nos indican que es esta diferencia en problemas de comunicación la que contribuye a la diferencia en la puntuación global entre los grupos de Lima y Piura. Si estos resultados pueden explicarse en función de procesos como los vínculos de apego o los estilos parentales percibidos, podría afirmarse que tales procesos influyen de manera diferente en las variables de problemas de comunicación en estas dos ciudades. De este modo, el mayor nivel de problemas de comunicación con ambos padres en Piura, respecto de Lima, posiblemente refleje algún elemento cultural, más dominante en algunas ciudades del interior del país que en la capital, respecto de la manera en que ambos padres consideran las reglas apropiadas tanto de comunicación como disciplinarias con sus hijos (tabla 4).

\section{Tabla 4}

\section{Prueba de Mann-Whithey de diferencias en comunicación padres- adolescentes entre los grupos de Lima y Piura}

\begin{tabular}{|c|c|c|c|c|c|}
\hline Variables & Grupo & Rango Promedio & $U$ & $Z$ & $P$ \\
\hline CGP & $\begin{array}{l}\text { Piura } \\
\text { Lima }\end{array}$ & $\begin{array}{c}120.92 \\
96.31\end{array}$ & 4503,000 & -2.898 & .004 \\
\hline CGM & $\begin{array}{l}\text { Piura } \\
\text { Lima }\end{array}$ & $\begin{array}{c}120.49 \\
96.73\end{array}$ & 4548,500 & -2.798 & .005 \\
\hline $\mathrm{ACP}$ & $\begin{array}{l}\text { Piura } \\
\text { Lima }\end{array}$ & $\begin{array}{l}110.85 \\
106.20\end{array}$ & 5580.500 & -.547 & .584 \\
\hline $\mathrm{ACM}$ & $\begin{array}{l}\text { Piura } \\
\text { Lima }\end{array}$ & $\begin{array}{l}114.24 \\
102.87\end{array}$ & 5217.500 & -1.340 & .180 \\
\hline PCP & $\begin{array}{l}\text { Piura } \\
\text { Lima }\end{array}$ & $\begin{array}{c}122.59 \\
94.67\end{array}$ & 4324.000 & -3.286 & .001 \\
\hline PCM & $\begin{array}{l}\text { Piura } \\
\text { Lima }\end{array}$ & $\begin{array}{c}124.62 \\
92.68\end{array}$ & 4107.000 & -3.759 & $<.001$ \\
\hline
\end{tabular}

Nota: $U=U$ de Mann-Whitney; $Z$ = Estadístico Z; CGP = puntuación total en comunicación, padre; $\mathrm{CGM}=$ puntuación total en comunicación; madre ACP = Apertura de comunicación, padre; $\mathrm{ACM}=$ Apertura de comunicación, madre; $\mathrm{PCP}=$ problemas de comunicación, padre; $\mathrm{PCM}=$ problemas de comunicación, madre

Fuente: elaboración propia

Los resultados indican en general mejores niveles de comunicación con la madre, lo cual indica probablemente una distinta socialización respecto a los papeles de género que los padres y madres atribuyen a sus hijos e hijas. Además, el padre suele estar más ausente (Rodrigo et al. 2005). También se debe considerar que la madre es quien tiene mayores responsabilidades 
en el cuidado y la crianza, en comparación con el padre. El estudio incide en la importancia de la calidad de la comunicación del adolescente con sus padres. Como señalaron Cámara et al. (2011), la familia es la mejor vía de soporte de sus miembros frente a la adversidad. La carencia de afectividad y de comunicación inciden en un deterioro del contexto global de la familia (Villavicencio \& Villarroel 2017). Todo ello es de particular importancia para los estudiantes universitarios, ya que, como se ha señalado, su vulnerabilidad no es poca, especialmente con la carga académica y financiera de hoy (Lee, 2020). Los resultados del estudio indican también, de manera indirecta, el posible papel de los factores culturales en las pautas de comunicación de cada figura parental (padre y madre) con sus hijos en la etapa tardía de la adolescencia.

\section{Conclusiones}

A nivel de la muestra total, la puntuación global en comunicación es similar respecto de ambas figuras parentales; sin embargo, se aprecia un nivel más elevado de apertura en relación con la madre y de problemas de comunicación frente al padre, lo cual corrobora los resultados observados en otros contextos culturales y geográficos.

Se observa un nivel similar en la comunicación global y en apertura de comunicación entre los universitarios de Lima y de Piura; sin embargo, el nivel de problemas de comunicación con ambas figuras parentales es más alto en el grupo de universitarios de Piura, lo que posiblemente refleje influencias de tipo cultural.

Es necesario ampliar la muestra a ciudades de la región andina (conocida en el Perú como la Sierra), lo cual sería beneficioso para ampliar las comparaciones interculturales del nivel de comunicación entre padres e hijos universitarios. Una limitación del trabajo es la no inclusión de la variable tipo de familia, dado que no solo existen las familias nucleares, sino también las familias monoparentales y la familia extensa.

\section{Referencias bibliográficas}

Apaza, C. M., Seminario, R. S., \& Santa-Cruz, J. E. (2020). Factores psicosociales durante el confinamiento por el Covid-19-Perú. Revista Venezolana de Gerencia, 25(90), 402-413. https://www. produccioncientificaluz.org/index. php/rvg/article/view/32385

Araujo, E., Ucedo, V. \& Bueno, R. (2018). Validación de la Escala de Comunicación Padres-Adolescente en jóvenes universitarios de Lima. Revista Digital de Investigación en Docencia Universitaria, 12(1), 253272. https://dx.doi.org/10.19083/ ridu. 12.56

Balluerka, N., Gómez, J., Hidalgo, M. D., Gorostiaga, A., Espada, J. A., Padilla, J. L., \& Santed, M. A. (2020). Las consecuencias psicológicas de la covid-19 y el confinamiento. Universidad del País Vasco. https:// www.ub.edu/web/ub/ca/menu eines/ noticies/docs/Consecuencias psicologicas COVID-19.pdf

Barneveld, H. O. Van. (2012). La percepción de la crianza en padres, madres e hijos adolescentes pertenecientes al mismo núcleo familiar. Liberabit. Revista de Psicología, 18(1), 75-81. https://bit. ly/3h0iFdR

Berbasé, R., Motrico, E. y Fuentes, MJ. (2001). Análisis psicométrico de dos escalas para evaluar estilos 
Araujo-Robles, Elizabeth Dany; Díaz Espinoza, Maribel; Díaz Mujica, Juana Yris Dinámica familiar en tiempos de pandemia (COVID -19): comunicación entre padres e hijos adolescentes universitarios

educativos parentales. Psicothema, 13 (4), 678-684. http://www. psicothema.com/psicothema. asp?id=496

Bradbury-Jones, C., \& Isham, L. (2020). The pandemic paradox: The consequences of COVID-19 on domestic violence. Journal of Clinical Nursing, 29(13-14), 2047-2049. https://doi.org/10.1111/jocn.15296

Broche-Pérez, Y., FernándezCastillo, E., y Reyes, D. (2020). Consecuencias psicológicas de la cuarentena y el aislamiento social durante la pandemia de COVID-19. Revista Cubana de Salud Pública, 46(supl.1). http://scielo.sld.cu/pdf/ rcsp/v46s1/1561-3127-rcsp-46s1-e2488.pdf

Brooks, S. K., Webster, R. K., Smith, L. E., Woodland, L., Wessely, S., Greenberg, N., \& Rubin, G. J. (2020). The psychological impact of quarantine and how to reduce it: rapid review of the evidence. The Lancet, 395(10227), 912-920. https://doi. org/10.1016/S0140-6736(20)30460$\underline{8}$

Cámara, A. M., \& Bosco-López. J. (2011) Estils d'educació a l'àmbit familiar. Revista Española de Orientación y Psicopedagogía 22(3), 257-276. https://www.raco. cat/index.php/EducacioSocial/article/ download/319834/428763/

Campbell, A. M. (2020). An increasing risk of family violence during the Covid-19 pandemic: Strengthening community collaborations to save lives. Forensic Science International: Reports, 2(April), 100089. https://doi. org/10.1016/j.fsir.2020.100089

Castañeda, R. C., Ochoa, G. M., Núñez Fadda, S. M., \& Callejas Jerónimo, J. E. (2019). Parentadolescent communication, psychological distress and attitude toward authority in Mexican adolescents: Their influence on school victimization. Estudios Sobre Educación, 36, 113-134. https://doi. org/10.15581/004.36.113-134

Caughlin, J., \& Ramey, M. (2005) The demand/withdraw pattern of communication in parent adolescent dyads. Personal Relationships, 12, 337-355. https://doi.org/10.1111/ j.1475-6811.2005.00119.x

Centre for Disease Control (2020). Helping Children Cope with Emergencies. https://www.cdc.gov/ childrnindisasters/helping-childrencope.html

CEPAL. (2020). América Latina y El Caribe ante la pandemia del covid-19. Efectos económicos y sociales. https://www.cepal.org/sites/ default/files/publication/files/45337/ S2000264 es.pdf

Crespo, J. (2011). Bases para construir una comunicación positiva en la familia. Revista de Investigación en Educación, 2(9), 91-98. https://dialnet.unirioja.es/servlet/ articulo?codigo $=4731297$

Cudris, L., Gutierrez, R., Barrios, A., Manjarres, M., \& Pérez, E. (2020). Comunicación familiar. Archivos Venezolanos de Farmacología $y$ Terapéutica, 39(3), 246-250. http:// saber.ucv.ve/ojs/index.php/rev aavft/article/view/19441

Dalton, L., Rapa, E., \& Stein, A. (2020). Protecting the psychological health of children through effective communication about COVID-19. The Lancet Child and Adolescent Health, 4(5), 346-347. https://doi. org/10.1016/S2352-4642(20)30097$\underline{3}$

Daniels, R., \& Rittenour, C.E. (2020). Reproducing Work and Family Norms through Daughter-Parent 
Communication. Journal of Social and Personal Relationships, 37(7), 23232341. https://journals.sagepub.com/ doi/full/10.1177/0265407520922912

Delfín, C., Cano, R., \& Peña, E. (2020). Funcionabilidad familiar como estrategia para la generación del emprendimiento social en México. Revista Venezolana de Gerencia, 25(89), 79-91. https://doi. org/10.37960/revista.v25i89.31387

Dorrance Hall, E., Scharp, K. M., Sanders, M., \& Beaty, L. (2020). Family communication patterns and the mediating effects of support and resilience on students' concerns about college. Family Relations, 69(2), 276-291. https://doi. org/10.1111/fare.12386

Duan, L., Shao, X., Wang, Y., Huang, Y., Miao, J., \& Yang, X. (2020). An investigation of mental health status of children and adolescents in china during the outbreak of COVID-19. Journal of Affective Disorders, 275, 112-118. https://doi.org/10.1016/j. jad.2020.06.029

Espada, J. P., Orgilés, M., Piqueras, J. A., \& Morales, A. (2020). Las buenas prácticas en la atención psicológica infanto-juvenil ante el COVID-19. Clínica y Salud, 31(2), 109-113. https://doi.org/10.5093/clysa2020a14

Garcés, M., Santoya, Y., \& Jiménez, J. (2020). Influencia de la comunicación familiar y pedagógica en la violencia escolar. Comunicar, 28(63),77-86. https://bit.ly/35VQaHY

Garrido, A., Reyes, A. G., Navarro, P., Orte, S., \& Navor, M. (2019). Comunicación familiar en estudiantes universitarios. Enseñanza e Investigación en Psicología, 1(2), 252-261.

Gomes, H. S., \& Gouveia-Pereira, M. (2019). Testing the general theory of crime with the circumplex model: curvilinear relations between family functioning and self-control. Deviant Behavior, 41(6), 779-791. https://doi. org/10.1080/01639625.2019.15964 $\underline{49}$

Gouveia-Pereira, M., Gomes, H., Miranda, M., \& Candeias, M.de J. (2020). Coesão e flexibilidade familiar: Validação do pacote FACES IV junto de adolescentes portugueses. Análise Psicológica, 38(1), 111-126. https://bit.ly/36a4Tzc

Guzmán, R., \& Pacheco, M. C. (2014). Comunicación familiar y desempeño académico en estudiantes universitarios. Zona Próxima, 20, 79-91. https://www.redalyc.org/ pdf/853/85331022008.pdf

Hernández, C., Valladares, A., Rodríguez, L., \& Selín, M. (2017). Comunicación, cohesión y satisfacción familiar en adolescentes de la enseñanza secundaria y preuniversitaria. Cienfuegos 2014. Revista de la Universidad Cienfuegos, 15, 341349. https://www.redalyc.org/articulo. oa? id=180051460009

Hsieh, K. Y., Wei, T. K., Dian, J. L., Wan, C. L., Kuan, Y. T., Wei, J. C., Li, S. C., Joh, J. H., Su, T. H., \& Chou, F. H. C. (2020). Mental health in biological disasters: From SARS to COVID-19. International Journal of Social Psychiatry. https://doi. org/10.1177/0020764020944200

Huarcaya, J. (2020). Consideraciones sobre la salud mental en la pandemia de COVID-19. Revista Peruana de Medicina Experimental y Salud Pública, 37(2), 327-334. https://doi. org/10.17843/rpmesp.2020.372.5419

Imran, N., Zeshan, M., \& Pervaiz, Z. (2020). Mental health considerations for children \& adolescents in COVID-19 pandemic. Pakistani Journal of Medical Sciences, 36, 
Araujo-Robles, Elizabeth Dany; Díaz Espinoza, Maribel; Díaz Mujica, Juana Yris

Dinámica familiar en tiempos de pandemia (COVID -19): comunicación entre padres e

hijos adolescentes universitarios

S67-S72. https://doi.org/10.12669/ pjms.36.COVID19-S4.2759

Kang, L., Li, Y., Hu, S., Chen, M., Yang, C., Yang, B. X., Wang, Y., Hu, J., Lai, J., Ma, X., Chen, J., Guan, L., Wang, G., Ma, H., \& Liu, Z. (2020). The mental health of medical workers in Wuhan, China dealing with the 2019 novel coronavirus. The Lancet Psychiatry, 7(3), e14. https://doi.org/10.1016/ S2215-0366(20)30047-X

Kapetanovic, S., \& Boson, K. (2020). Discrepancies in parents' and adolescents' reports on parentadolescent communication and associations to adolescents' psychological health. Current Psychology. doi: https://doi. org/10.1007/s12144-020-00911-0

Kapetanovic, S., Rothenberg, W. A., Lansford, J. E., Bornstein, M. H., Chang, L., Deater-Deckard, K., Di Giunta, L., Dodge, K. A., Gurdal, S., Malone, P. S., Oburu, P., Pastorelli, C., Skinner, A. T., Sorbring, E., Steinberg, L., Tapanya, S., Uribe Tirado, L. M., Yotanyamaneewong, S., Peña Alampay, L., ... Bacchini, D. (2020). Cross-cultural examination of links between parent-adolescent communication and adolescent psychological problems in 12 cultural groups. Journal of Youth and Adolescence, 49(6), 1225-1244. https://doi.org/10.1007/s10964-02001212-2

Kilincel, S., Kilincel, O., Muratdagi, G., Aydin, A., \& Usta, M. B. (2020). Factors affecting the anxiety levels of adolescents in home-quarantine during the COVID-19 pandemic in Turkey. Asia Pac Psychiatry, e12406. https://doi.org/10.1111/appy.12406

Koushik, N. S. (2020). A population mental health perspective on the impact of COVID-19. Psychological Trauma: Theory, Research, Practice, and Policy, 12(5), 529-530. http:// dx.doi.org/10.1037/tra0000737

Lee, J. (2020). Mental health effects of school closures during COVID-19. The Lancet Child and Adolescent Health, 4(6), 421. https://doi. org/10.1016/S2352-4642(20)30109$\underline{7}$

Lippi, G., Henry, B. M., Bovo, C., \& Sanchis-Gomar, F. (2020). Health risks and potential remedies during prolonged lockdowns for coronavirus disease 2019 (COVID-19). Diagnosis (Berlin, Germany), 7(2), 85-90. http:// doi.org/10.1515/dx-2020-0041

Liu, Q., Lin, Y., Zhou, Z., \& Zhang, W. (2019). Perceived parentadolescent communication and pathological internet use among Chinese adolescents: a moderated mediation model. Journal of Child and Family Studies, 28, 1571-1580. https://doi.org/10.1007/s10826-01901376-X

Lozano, A., Fernández-Prados, J., Figueredo, V. \& Martínez, A. M. (2020). Impactos del confinamiento por el covid-19 entre universitarios: satisfacción vital, resiliencia y capital social online. International Journal of Sociology of Education, 79. Special issue (COVID-19: Crisis and Socioeducative Inequalities and Strategies to Overcome Them), 79-104. https://doi.org/10.17583/ rise. 2020.5925

Moretti, M.P., Lechuga, M.J. \& Torrecilla, N.M. (2020) Desarrollo Psicomotor en la infancia temprana y funcionalidad familiar. Psychologia, 14(2), 37-48. https:// doi.org/10.21500/19002386.4646

Oliva, A., Parra, A. \& Sánchez-Queija, I. (2002). Relaciones con padres e iguales como predictoras del ajuste emocional y conductual durante la adolescencia. Apuntes de Psicología, 
20, 3-16.

Olson, D. H., \& Gorall, D. M. (2003). Circumplex model of marital and family systems. En F. Walsh (Ed.), Normal family processes (3ra. Ed.) (pp. 514-547). Nueva York: Guilford.

Olson, D. H., Sprenkle, D. H., \& Russell, C. S. (1979). Circumplex model of marital and family systems I: Cohesion \& adaptability dimensions, family types and clinical applications. Family Process, 18, 3-28.

Olson, D. H., Waldvogel, L., \& Schlieff, M. (2019). Circumplex model of marital and family systems: An update. Journal of Family Theory \& Review, 11, 188-211. https://doi.org/10.1111/ jftr.12331

Pastor, J. C. (2020). Los efectos psicológicos del Covid-19. Archivos de la Sociedad Española de Oftalmología, 95(9), 417-418. https:// doi.org/10.1016/j.oftal.2020.06.010 _

Pérez, M., \& Alvarado, C. (2015). Los estilos parentales: Su relación en la negociación y el conflicto entre padres y adolescentes. Acta de Investigación Psicológica, 5(2), 1972-1983. https://doi.org/10.1016/ s2007-4719(15)30017-x

Pinquart, M. (2017). Associations of parenting dimensions and styles with externalizing problems of children and adolescents: An updated meta-analysis. Developmental Psychology, 53(5), 873-932. https:// doi.org/10.1037/dev0000295

Polit, D. F. \& Beck, C. T. (2012). Nursing research. Generating and assessing evidence for nursing practice (9na. Ed.). Filadelfia: Lippincott Williams \& Wilkins.

Prime, H., Wade, M., \& Browne, D. T. (2020). Risk and resilience in family well-being during the COVID-19 pandemic. American Psychologist, 75(5), 631-643. $\quad$ https://doi. org/10.1037/amp0000660

Raimundi, M., Molina, M., Leibovich, N., \& Schmidt, V. (2017). La comunicación entre padres e hijos: su influencia sobre el disfrute $y$ el flow adolescente. Revista de Psicología, 26(2), 1-14. https://doi. org/10.5354/0719-0581.2017.46881

Rivera, R., \& Cahuana M. (2016). Influencia de la familia sobre las conductas antisociales en adolescentes de Arequipa-Perú. Actualidades en Psicología, 30(120), 85-97. http://dx.doi.org/10.15517/ ap.v30i120.18814

Rodrigo, M. J., García, M., Máiquez, M. L., \& Triana, B. (2005) Discrepancies between parents and adolescent children in the perceived frequency and emotional intensity in family conflicts. Estudios de Psicología, 26(1), 21-34. https://doi. org/10.1174/0210939053421407

Ruvalcaba, N., Orozco, M., Gallegos, J. \& Nava, J. M. (2018). Relaciones escolares, comunicación con padres y prosocialidad como predictores de emociones positivas. Liberabit: Revista Peruana de Psicología, 24(2), 183-93. https://bit.ly/2Szvltf

Santos, J. (2019). Comunicación en familias nucleares con hijos adolescentes. Revista Científica Digital de Psicología, 8, 1-11.

Saurabh, K., \& Ranjan, S. (2020). Compliance and psychological impact of quarantine in children and adolescents due to Covid-19 pandemic. The Indian Journal of Pediatrics. https://doi.org/10.1007/ s12098-020-03347-3

Serafini, G., Parmigiani, B., Amerio, A., Aguglia, A., Sher, L., \& Amore, M. (2020). The psychological impact 
Araujo-Robles, Elizabeth Dany; Díaz Espinoza, Maribel; Díaz Mujica, Juana Yris Dinámica familiar en tiempos de pandemia (COVID -19): comunicación entre padres e hijos adolescentes universitarios

of COVID-19 on the mental health in the general population. QJM: An International Journal of Medicine, 113(8), 529-535.

Simpson, E. G., Lincoln, C. R., \& Ohannessian, C. M. C. (2020). Does adolescent anxiety moderate the relationship between adolescentparent communication and adolescent coping? Journal of Child and Family Studies, 29(1), 237-249. https://doi.org/10.1007/s10826-019$\underline{01572-9}$

Torales, J., O'Higgins, M., CastaldelliMaia, J. M., \& Ventriglio, A. (2020). The outbreak of COVID-19 coronavirus and its impact on global mental health. International Journal of Social Psychiatry, 66(4), 317-320. https:// doi.org/10.1177/0020764020915212

Turkdogan, T., Duru, E., \& Balkis, M. (2019). Circumplex model of family functioning in Turkish culture: Western family systems model in a Eurasian country. Journal of Comparative Family Studies, 50(2), 184-199. https://doi.org/10.3138/ jcfs.50.2.005

Usher, K., Bhullar, N., Durkin, J., Gyamfi, N., \& Jackson, D. (2020). Family violence and COVID-19: Increased vulnerability and reduced options for support. International Journal of Mental Health Nursing, 29(4), 549-552. https://doi.org/10.1111/ inm.12735

Van Dijk, M. P. A., Branje, S., Keijsers, L., Hawk, S. T., Halle, W. W., \& Meeus, W. (2014). Self-concept clarity across adolescence: Longitudinal associations with open communication with parents and internalizing symptoms. Journal of Youth and Adolescence, 43, 1861-1876. https://doi.org/10.1007/ s10964-013-0055-x
Velásquez, L., y Salom, R. (2008). La comunicación interpersonal dentro de la dinámica familiar ante el reto de la adopción. TELOS. Revista de Estudios Interdisciplinarios en Ciencias Sociales, 10(1), 122 - 138. http://ojs.urbe.edu/index.php/telos/ article/view/1350/1243

Villavicencio, C. E., \& Villarroel, M. F. (2017). Comunicación afectiva en familias desligadas. Fides et Ratio, 13(13), 15-39. https://bit.ly/3vZ27Hf

Wang, C., Pan, R., Wan, X., Tan, Y., Xu, L., Ho, C. S., \& Ho, R. C. (2020). Immediate psychological responses and associated factors during the initial stage of the 2019 Coronavirus disease (COVID-19) epidemic among the general population in China. International Journal of Environmental Research and Public Health, 17(5), 1729. https://doi. org/10.3390/ijerph17051729

Zhou, S. J., Zhang, L. G., Wang, L. L., Guo, Z. C., Wang, J. Q., Chen, J. C., Liu, M., Chen, X., \& Chen, J. X. (2020a). Prevalence and socio-demographic correlates of psychological health problems in Chinese adolescents during the outbreak of COVID-19. European Child and Adolescent Psychiatry, 29(6), 749-758. https:// doi.org/10.1007/s00787-020-01541$\underline{4}$

Zhou, X., Zhen, R., \& Wu, X. (2020b). Insecure attachment to parents and PTSD among adolescents: the roles of parent-child communication, perceived parental depression, and intrusive rumination. Development and Psychophatology. https://doi. org/10.1017/S0954579420000498 